\title{
Research on "Three in One" Combination of Innovation Models of Medical Student Professional Education, Humane Quality Education and Medical Ethics Education on Improving Medical Ethics and Establishing a Harmonious Doctor-Patient Relationship
}

\author{
Juning Wang \\ School of Medicine, Xi'an Peihua University, Xi'an, Shaanxi, 710125
}

Keywords: Three in One; innovation models; doctor-patient relationship

\begin{abstract}
With the continuous deepening reform of the medical system and people's growing legal awareness, the community's requirements for the medical industry are gradually increasing. In recent years, the number of medical disputes has increased significantly, and the relationship between doctors and patients has become increasingly tense. It has gradually become a social problem that needs to be resolved. Faced with this situation, hospitals and medical personnel should start from their own, change their concepts in a timely manner, standardize the behavior of medical services, strengthen communication between doctors and patients, increase mutual understanding, resolve conflicts between doctors and patients, and jointly create a civilized medical environment to effectively safeguard the interests of both parties, and to promote the harmonious development of society. How to build a harmonious and healthy doctor-patient relationship is a major issue worthy of study by all walks of life, especially hospital administrators.
\end{abstract}

\section{Introduction}

From the beginning of its development to the present, the development trend of medical technology and life science has always been strong. Despite the magnificent changes in contemporary medicine, the relationship between doctors and patients has never stopped, and the realization of a harmonious doctor-patient relationship has also become a life ethics. The eternal pursuit of learning. In recent years, the contradictions between doctors and patients have become more and more severe. They have constantly presented new ethical and social challenges to medical personnel. At the same time, the ethical issues between doctors and patients are so new, unique and different, how to deal with these ethical challenges and make medical ethics Research results and theories have been translated into actions in time through systems and guidelines. Effectively alleviating the current contradictions between doctors and patients and advancing the process of building a harmonious doctor-patient relationship have become the core issues and values of bioethics research.

\section{The Origin of the Conflict between Doctors and Patients}

Doctor-patient relationship is a unique social relationship in human culture. With the development of economic and social medical care, doctor-patient conflicts can be divided into target-based doctor-patient conflicts, cognitive doctor-patient conflicts, and emotional doctor-patient conflicts, regardless of type. The conflict between doctors and patients is always closely related to many factors in society, such as legal, institutional, and cultural factors, and thus has many root causes.

Modern medical activities are inseparable from the law. Medical practice according to law has become a basic requirement for medical activities. The essence of practicing medicine in accordance with the law is to ensure the realization of the rights and obligations of both doctors and patients. At present, some legal provisions in China are not perfect, which is precisely the external source of conflict between doctors and patients. The rights and obligations of both doctors and 
patients have the unity of opposites, presuppose each other and restrict each other. When the rights and obligations of both doctors and patients cannot be coordinated, they will evolve into conflicts. For example, whether the right to know and the obligation to communicate are unanimous, often determining whether the doctor-patient relationship is harmonious. As medical personnel, they have the right to understand the "privacy" of the patient. This is a prerequisite for correctly diagnosing the disease and treating the disease. At the same time, as a patient, he has the right to know what disease he has and he has the right to know the treatment plan. If doctors and patients perform their duties, the relationship between doctors and patients will be harmonious. If doctors and patients have problems with one side, both doctors and patients will conflict and even confront each other. Some existing legal systems have foreshadowed the doctor-patient conflict. Basically, the law is to maintain a good social order and to build a good doctor-patient relationship. In practice, whether the law can achieve legislative goals is always subject to the social environment at that time. For example, the provision for the reversal of the burden of proof in medical disputes is based on the information asymmetry between the doctor and the patient and the unequal status. The purpose of the legislation is to protect the vulnerable position of the patient from the legal point of view. The result of the law has led to the defense. Derivatives of sexual health, excessive rights protection and other phenomena.

\section{The Adverse Effects of Tension between Doctors and Patients}

Nervous doctor-patient relationship, lack of trust and cooperation in the diagnosis and treatment process, will inevitably affect the therapeutic effect, and this status often leads to the emergence of a vicious circle. When doctors and patients develop contradictions and disputes, medical institutions have to invest a great deal of manpower and time to deal with disputes. The normal work order has been disrupted, which has greatly affected the daily treatment work. The credibility of medical institutions has been questioned. The development of medicine is inseparable from exploration and experimentation. In the face of the increasingly tense relationship between doctors and patients, and increasingly intensified medical disputes, doctors are no longer thinking of trying their best to eradicate the disease. Instead, they are increasingly conservative in their adherence to the inherent and mature medical measures. It is difficult to have the courage and courage to innovate and exceed. . The medical dispute caused by the tense doctor-patient relationship has not only been affected by the medical institutions and the patients and their families. The scope of violence and conflict has been gradually expanded, and the personnel involved have become increasingly complex, becoming a social discomfort that is widely concerned by the general public. .

Constructing harmonious doctor-patient relationship is conducive to enriching and perfecting the theory of building a harmonious society. The effect of medical treatment depends on the ethical quality and professional skills of medical personnel, and depends on the degree to which the medical resources are perfect and adequate, and on the level of patient satisfaction. Of course, the medical effects will also be affected by the relationship between doctors and patients. A harmonious relationship between doctors and patients will help promote the professional level of medical personnel and assist the treatment process, thereby promoting the improvement of the quality of medical services; conflicting doctor-patient relationship The restraint of the normal level of medical personnel affects the overall therapeutic effect. How to build a harmonious doctor-patient relationship is not only a question of interests between doctors and patients, but also more importantly reflects the ethical and moral issues of doctors and patients. However, it is well known that medical and health services are one of the important social and public products. Then, Harmonious doctor-patient relationship is inevitably an important part of a harmonious society. Therefore, a comprehensive analysis and multi-perspective study of the theory of building a harmonious relationship between doctors and patients is a fulfillment of the theory of building a harmonious socialist society. 


\section{Harmonious Construction of Doctor-Patient Relationship}

The development of modern society and the enhancement of the sense of rights have caused various groups, organizations or individuals to inevitably conflict with others in the pursuit of individual rights. In the doctor-patient relationship, this conflict of rights is particularly evident. All along, many scholars who have researched doctor-patient relationship have focused doctor-patient disputes on conflicts of interest. This view confuses the relationship between conflicts of interest and rights conflicts. In fact, the conflict of interest is merely an appearance. It is essentially a doctor-patient relationship. Conflicts between rights. Therefore, doctor-patient conflict is an outbreak of doctor-patient conflicts in contemporary society. It is a conflict of interests, and it is also a conflict of rights. The unclear definition of rights between doctors and patients and the disorder of borders are important causes of the conflict between doctors and patients. To resolve the conflicts between doctors and patients, we need to redefine the boundaries of the rights of both doctors and patients. We must also respect the rights of doctors while respecting and guaranteeing the rights of patients. On the other hand, we must also prevent the exercise of rights and the abuse of rights. It is necessary to clarify the rights and obligations of the doctors and the patients through legislation, balance the doctor-patient relationship, and resolve the conflict between doctors and patients. The introduction of the "Regulations on the Prevention and Handling of Medical Disputes in Jiangxi Province", "Regulations on the Management of Medical Institutions in Yunnan Province", and "The Medical Regulations on Shenzhen Special Economic Zone" have effectively promoted the process of medical legislation.

The intensification of conflict between doctors and patients has a great destructive effect on the normal medical order and doctor-patient relationship. The theory of social conflict points out that "safety valve" is a safety mechanism that maintains social operation and maintains social relations. A good social safety valve mechanism is essential to control the doctor-patient conflict. In view of the conflict between doctors and patients, in the theory and practice should be committed to building a multi-level, multi-form social security valve mechanism, so as to achieve the purpose of building a harmonious doctor-patient relationship. It is necessary to actively resolve conflicts through system construction and rule reconstruction, introduce the role of medical social workers, and reconcile the direction of harmonization between doctors and patients. In addition to the existing mechanisms for handling disputes among doctors and patients through negotiation, administrative mediation, and civil litigation, we will establish a third-party mediation agency and third-party arbitration agency that accepts, recognizes, mediates, and arbitrates all parties involved in the doctor-patient conflict to maximize The subjective interference of various stakeholders, playing authoritative, professional and people-oriented. The state adopts legislation to establish compulsory medical damage compensation insurance. The parties involved in compulsory medical activities purchase insurance from the insurance company. The insurance company is responsible for compensation, which is conducive to the ease of doctor-patient relationship.

Under the new historical conditions, the conflict between doctors and patients shows more complicated characteristics, objectively analyzes the current situation of doctor-patient conflicts, and explores the deep social psychological causes in many dimensions, and proposes scientific and effective solutions accordingly, thereby building harmony. The doctor-patient relationship. In-depth study of the causes of conflict between doctors and patients, you can use social and psychological analysis to retrieve the lost integrity. The majority of medical disputes stem from the lack of integrity of doctors and patients, self-centeredness, over-emphasis on economic interests, and lack of humane care. Therefore, we must focus on nurturing and practicing the unified core values of the entire society, reconstruct the social moral trust mechanism, and establish a sound credit moral self-conscious system, so that both the doctor and the patient can become a moral community that holds common value appeals; Medical and health management departments and medical institutions Medical ethics management should be incorporated into the whole process of hospital management, establish a hospital medical ethics management committee, create a good practice environment, so that honesty and trustworthiness become self-discipline, consciously abide by the behavior 
standards.

Examining the tensions in the current doctor-patient relationship in China from the perspective of unbalanced interests, we will find that the vast majority of medical disputes are in essence the imbalance of benefits between doctors and patients. To realize the harmony between doctors and patients and realize the dynamic balance between doctors and patients, it is necessary to establish a rule system for medical cultivation, increase health investment, strengthen the construction of the health legal system, establish an effective doctor-patient communication mechanism, consultation mechanism for doctors and patients, and doctors Benefit protection mechanism, smooth channels for doctors and patients to express their interests, properly resolve contradictions between doctors and patients, and construct a set of effective guarantee mechanisms to achieve a harmonious relationship between doctors and patients from internal guarantees and external guarantees. Both doctors and patients are in fact a community of great destiny. The common enemy is disease, and there is a natural balance between doctors and patients, and there is also a natural balance. Establishing legal rights and obligations between doctors and patients, establishing a modern relationship between doctors and patients, and morally rebuilding trust between doctors and patients to prevent conflicts between doctors and patients, so that many rights and obligations are truly balanced, and doctor-patient conflicts are realized. The dissolution and transformation of the disease will promote the harmonious compatibility of doctor-patient relationship.

\section{Conclusion}

The medical staff thought that their own work was relatively perfect and had no significant impact on the doctor-patient relationship. Relatively speaking, the descriptive statistics show that the medical staff has insufficient attention to the patient's psychological condition and insufficient psychological counseling. In this regard, medical workers should have a strong concept and ability of communication between doctors and patients, allowing patients to understand the high-risk and uncertainties of the medical service industry, and at the same time formulate treatment and care plans in a targeted way to alleviate anxiety and tension of patients. The psychology thus promotes the harmonious development of doctor-patient relationship.

\section{Acknowledgements}

Xi'an Peihua College's 2018 year school-level counselor work special research project, project name: constructing "three-in-one" study of Innovative Medical Education Model, Item Number: PHSZ201816

\section{References}

[1] Shi Yuhong. Analysis of the causes of medical disputes that affect social harmony and stability and countermeasures [J]. Journal of Shanxi University of Finance and Economics, 2015(4): 171172.

[2] Gao Xiang. How the United States deals with doctor-patient relationship [J]. Southern Weekend, 2012 - 8 - 26.

[3] Wang Shichuan. To reconstruct the trust of doctors and patients must be broken from the system [J]. Forum of Chinese Party and Government Officials, 2014 (5): 78.

[4] Ruan Guoci. Research on doctor-patient relationship from the perspective of social conflict theory [J]. Jianghan Forum, 2014 (3): 140 - 144.

[5] Guo Ning. Construction of Harmonious Relationship between Doctors and Patients with Party Building Work [J]. Hospital Management Forum, 2013(31):24-26. 